\title{
Complete posterior migration of intact vertebral body in spinal tuberculosis
}

\author{
Krishnamurthy Sridhar, Prasad Krishnan
}

Department of Neurosurgery, National Neurosciences Center, Calcutta, India

\author{
Address for correspondence: \\ Dr. K. Sridhar, \\ 15 MGR Road, Kalakshetra Colony, \\ Besant Nagar, Chennai, India. \\ E-mail: neurosridhar@rediffmail.com
}

DOI: $10.4103 / 0028-3886.55600$

\begin{abstract}
Spinal tuberculosis most commonly presents as a paradiscal lesion involving the disc space and adjacent vertebral bodies. Atypical forms of spinal tuberculosis have been described and are most often a result of posterior element involvement. The authors report a patient, who presented with complete posterior migration of an intact vertebral body, a complication of spinal tuberculosis that has not been reported till date. A 12 -year-old girl with history of pulmonary tuberculosis presented with progressive paraparesis and back pain. Plain X-rays and MRI revealed that the L2 vertebral body had migrated posteriorly into the spinal canal, without significant movement of the posterior elements. The vertebral body was normal, with no erosion or bone loss. However, bilateral pedicle and facet joint involvement was seen. The neural elements were decompressed through an anterolateral retroperitoneal approach and the spine reconstructed. The authors present this rare manifestation of spinal tuberculosis and discuss the possible mechanisms of this presentation.
\end{abstract}

Key words: Migration, posterior elements, spinal tuberculosis

\section{Introduction}

Classically four types of involvement of the spine have been described in spinal tuberculosis, with the paradiscal variety being the most commonly observed in practice. ${ }^{[1]}$ The anterior, central, and appendiceal varieties are seen less frequently. Radiologically, while different types of spinal involvement have been described, with advanced disease, involvement of the vertebral body with a fragmentary type of osseous destruction, collapse, sequestra formation have been thought mandatory. ${ }^{[2]}$ Complete posterior migration of an intact vertebral body in tuberculosis spine, has till date, not been described in the literature. The authors report such a case and discuss the probable pathogenesis of such a migration.

\section{Case Report}

A 12-year-old girl was admitted with mid-back pain of one-month duration, inability to move both her lower limbs and a decrease in sensations of her legs for five days, and incontinence of urine and motion of four days duration. She was diagnosed with pulmonary tuberculosis four months earlier and was started on a 3 drug regime of antitubercular drugs (ATD). She was diagnosed to have spinal tuberculosis a month before this admission because of her back pain, when she was advised complete bed rest and continuation of ATD by her physician. There were no cranial or upper limb symptoms. On examination, she was an anemic and malnourished child. Her higher intellectual functions, cranial nerves, and upper limbs were normal. She had flaccid paraplegia below L2 with absent reflexes. Anal sphincter was lax. While there were absent sensations below the L3 dermatome on the right, there was preservation of approximately $10 \%$ on the left. There was a tender palpable gibbus at the level of L2.

Plain radiographs done 45 and 15 days prior to admission showed progressive posterior migration of an intact L2 vertebral body [Figures 1 and 2]. The cortical margins of the body appeared intact, while the pedicles of L2 were not seen bilaterally. MRI of the spine on admission showed complete posterior migration of the intact L2 vertebral body causing extradural thecal compression [Figure 3]. The adjacent discs appeared intact and were occupying the space created by the migrating body. Prevertebral and paravertebral fluid collections were present. 


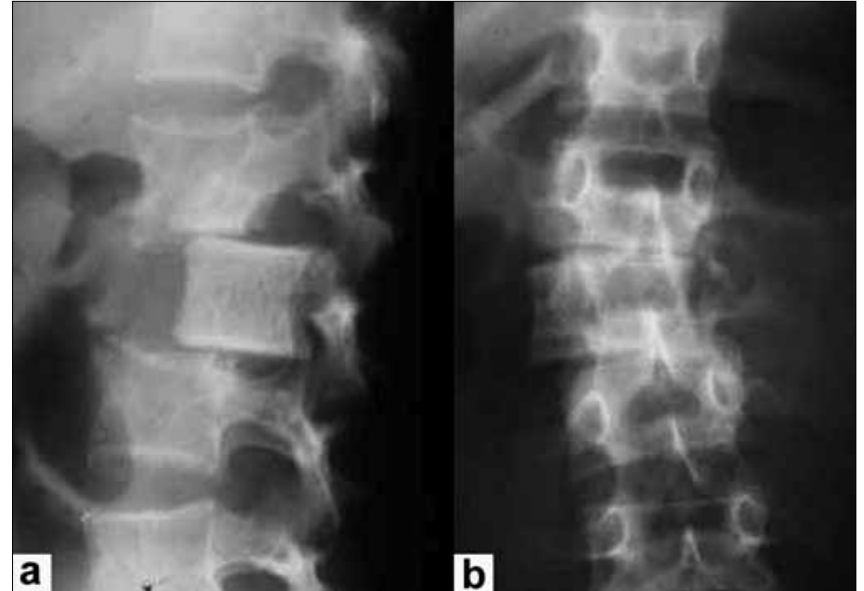

Figure 1: Plain X-rays of the lumbar spine lateral view (a) antero-posterior view; (b) taken at $\mathbf{4 5}$ days prior to admission showing posterior displacement of an intact $L 2$ vertebral body with bilateral destruction of the pedicles and pars

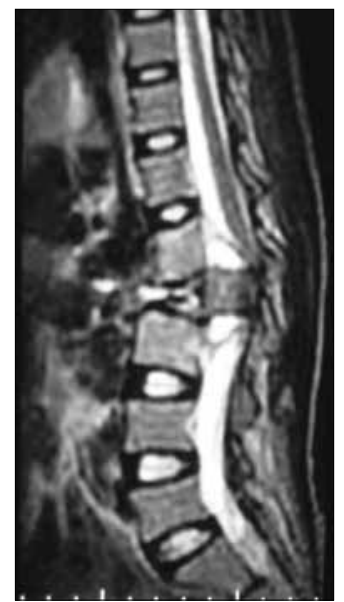

Figure 3: MRI T2 weighted sagittal image showing the complete posterior migration of the $L 2$ vertebral body

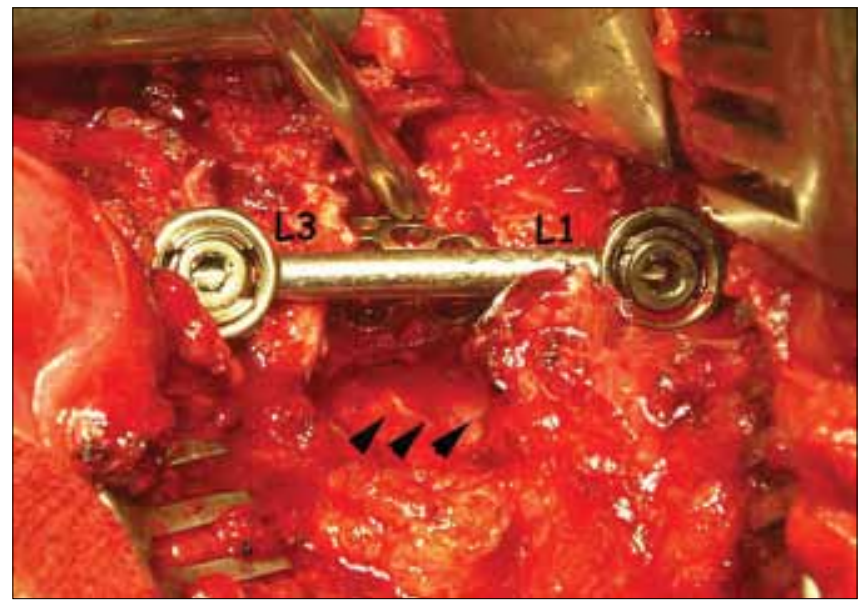

Figure 5: Intraoperative photograph showing the decompressed dura [arrowheads] and the reconstruction of the spinal column

The child was operated upon by a retroperitoneal approach. Intraoperatively the $\mathrm{L} 2$ body was found intact and retropulsed into the spinal canal. [Figure 4] The

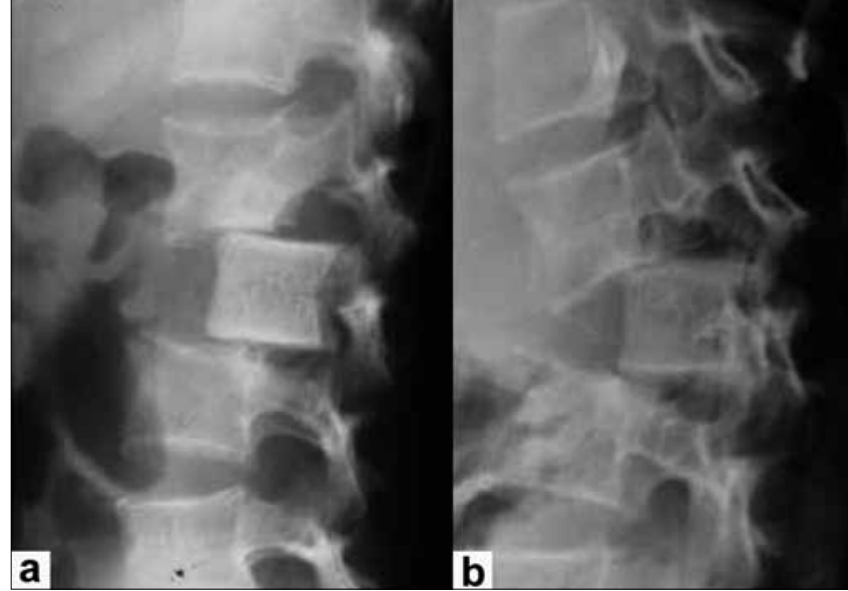

Figure 2: Plain X-rays of the lumbar spine lateral view taken (a) 45 days (b) 15 days prior to admission showing progressive posterior migration of the intact $L 2$ body

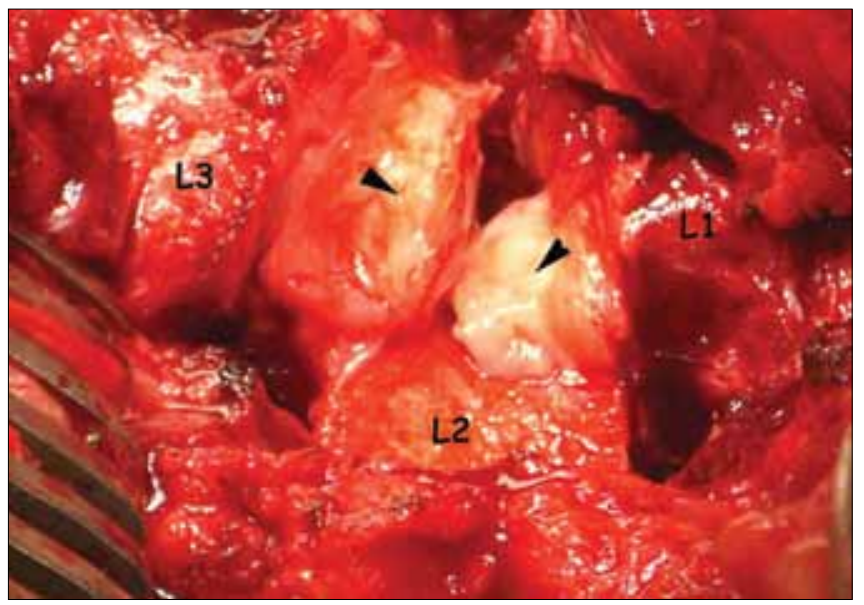

Figure 4: Intraoperative photograph showing the complete posterior migration of the L2 vertebral body. The adjacent discs [arrowheads] appear normal but were filled with caseous material

adjacent disc spaces were full of infected caseous material. The L2 body appeared normal and was excised and the dura decompressed. Caseous material was evacuated from the prevertebral and the paravertebral regions. Once the dura was decompressed, the spine was reconstructed using a titanium cage filled with autologous cancellous bone, and a screw-rod system [Figures 5 and 6]. The child was continued on a 5 drug ATD regime. She was mobilized after two weeks on a thoracolumbar brace. With physiotherapy she is gradually improving, but still unable to stand without support.

\section{Discussion}

The diagnosis of tuberculosis of the spine is based on clinical and radiological features. Clinical features of back pain, paraspinal muscle spam and a gibbus, in endemic areas, are signs of tuberculous spinal disease unless proved otherwise. Narrowing of the disc space with erosion of the paradiscal regions of adjacent vertebral 


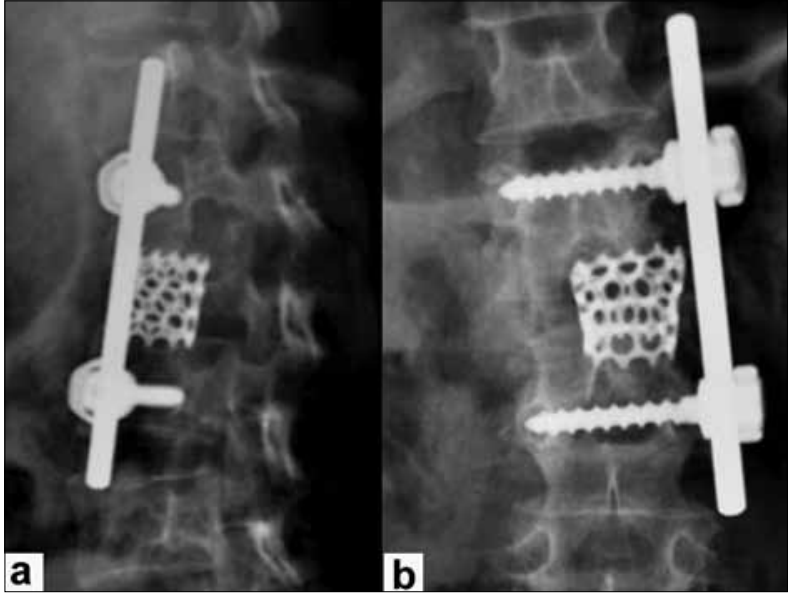

Figure 6: Post-operative X-rays (a) lateral; (b) antero-posterior views showing the reconstruction of the spinal column and implants in position

bodies is often the initial and the most common finding on plain X-rays and reflects paradiscal type of disease. ${ }^{[2]}$ As the disease progresses, bone loss and kyphosis develop. Less common patterns of spinal tuberculosis are the central, the anterior and the appendiceal types.

The appendicular type of spinal tuberculosis is not as common as the other types which primarily involve the vertebral bodies or the anterior elements of the spinal column. Isolated involvement of the posterior elements has been documented in only $3.06 \%$ of the 1,076 patients with spinal tuberculosis reviewed by Narlawar et al..$^{[3]}$ The exact incidence may be as high as 5 and 10\% in endemic areas. ${ }^{[4]}$ The pedicles and laminae are the most commonly involved portions of the posterior elements usually both being involved. Isolated pedicle involvement was seen in 12 of the 27 cases reported by Kumar. ${ }^{[4]}$ Bilateral pedicle involvement was reported in six out of the twenty-four patients by Narwalkar et al. ${ }^{[3]}$ Kyphosis and spinal deformity is uncommon in posterior spinal disease as spinal stability is not grossly affected. Gross spinal instability and devastating neurological deficit occur when there is a complex and bilateral involvement of the joints and of the middle column.

In the present case, the clinical signs are classical of spinal tuberculosis. Radiologically, the intact L2 vertebral body was seen to have migrated posteriorly and the MRI confirmed its complete displacement into the canal. On imaging, there was no feature suggestive of erosion, sclerosis, destruction or marrow changes in the displaced L2 body. To the best of our knowledge such finding of a posterior migration of an intact vertebral body secondary to tuberculosis has not been described till date. Gandhi et al., reported a case of spinal tuberculosis with lateral migration of the L4 vertebral body. ${ }^{[5]}$ In their case, the said patient presented with backache and fever. Radiology showed the L4 body lying laterally in the paraspinal gutter within a psoas abscess. Though the presence or absence of erosion of the L4 body has not been commented upon by the authors, the image suggests erosion of the body.

For migration of vertebral body, without displacement of the posterior elements, it is necessary that it is "freed" bilaterally from the pars articularis. Nonvisualization of both pedicles of L2 on the initial X-ray in our patient, indicates the probable initial destruction of the pars and the articular facets bilaterally. The intraoperative finding of both adjacent disc spaces filled with caseous material indicates the spread of infection anteriorly leading to laxity of the adjacent annular attachments of discs, allowed the vertebral body to migrate. Why the body migrated posteriorly and not anterior, is probably because, with an initial posterior element infection, the posterior ligaments became lax before the anterior ligaments. This could have started the posterior dislocation of the body, which then progressed to a complete migration. Chadha et al., reported three cases of tuberculosis co-existing with spondylolisthesis at the lumbosacral region. ${ }^{[6]}$ It was opined that the tubercular process involved the posterior elements and caused secondary spondylolisthesis.

\section{Conclusion}

Complete posterior migration of an intact vertebral body into the spinal canal in tuberculous spine, is a rare phenomenon not described earlier in the literature. This could result from involvement of the ligaments holding the vertebra and destruction of the bilateral pars and facets. It is essential, therefore, to review the images carefully and follow the progress of the disease in order to recognize the involvement of various spinal elements. This is important as the pathology of the disease may progress to destabilize the spinal columns resulting in devastating neurological deficits. Thus, aggressive treatment and avoiding instability is the key to a complete recovery.

\section{References}

1. Sridhar K. Tuberculosis of the spine. In: Ramamurthi B, Tandon PN Editors. Textbook of Neurosurgery. $2^{\text {nd }}$ ed. New Delhi: BI Churchill Livingstone; 1996. p. 498-513.

2. Joseffer SS, Cooper PR. Modern Imaging of spinal tuberculosis. J Neurosurg Spine 2005;2:145-50.

3. Narlawar R, Shah, JR, Pimple MK, Patkar, DP, Patankar T, Castillo M. Isolated tuberculosis of the posterior elements of the spine: Magnetic resonance imaging findings in 33 patients. Spine 2002;27:275-81.

4. Kumar K. A clinical study and classification of posterior spinal tuberculosis. Int Orthop 1985;9:147-52.

5. Gandhi D, Sharma R, Kotwal PP, Hatimota P. Migrating lumbar vertebra. Lancet 2000;356:1485.

6. Chadha M, Agarwal A, Kumar S. Spinal tuberculosis with concomitant spondylolisthesis: Coexisting entities or 'cause and effect'? Spinal Cord 2006;44:399-404.

Accepted on 02-07-2009

Source of Support: Nil, Conflict of Interest: None declared. 